\title{
Architecture of a MOOC based on CourseBuilder
}

\author{
Pedro Pernías Peco \\ Department of Software and Computing Systems \\ University of Alicante \\ Alicante, Spain \\ Email: p.pernias@ua.es
}

\author{
Sergio Luján-Mora \\ Department of Software and Computing Systems \\ University of Alicante \\ Alicante, Spain \\ Email: sergio.lujan@ua.es
}

\begin{abstract}
This paper focuses on the development of UniMOOC aemprende, a MOOC (Massive Open Online Course) based on Google CourseBuilder. Firstly, we discuss the current available platforms aimed to develop MOOCs: before developing UniMOOC aemprende, different MOOC platforms were reviewed in order to choose the most suitable for our requirements. Then, we explain why we selected Google CourseBuilder as the most suitable option for the requirements of our MOOC. Finally, we present the architecture of our MOOC, composed of different components that engage our students and help them succeed.
\end{abstract}

Keywords-MOOC; LMS; e-learning; online learning; distance learning; architecture

\section{INTRODUCTION}

MOOCs (Massive Open Online Courses) have been around us since 2008, when around 2,300 students took part in a course called "Connectivism and Connective Knowledge", organized by the University of Manitoba (Canada). However, 2012 was widely recognized as "The year of the MOOC" [1], because some MOOC initiatives, such as Coursera, Udacity, or edX, gained a world-wide popularity. Many experts consider MOOCs a "revolution in education" [2]. However, other experts think is too soon to make such a claim and MOOCs still have to prove their real value [3].

So far, MOOCs do not differ much from online courses that have existed among us for years: a syllabus (with course objectives and expected outcomes), a calendar, some educational materials (mainly videos, but also lecture notes and assigned readings), some activities or projects, some quizzes and exams (usually multiple choice exercises) to assess students' learning, and a forum to discuss with the teacher and other students.

Coursera, edX, and Udacity form the triumvirate of major MOOC providers. All of them have continued to grow since their birth, offering new courses every month. Unfortunately, they are turning into an "exclusive club", because not all higher education institutions can offer their MOOCs through those MOOC providers. Therefore, the interest on how to offer a MOOC using its own means is increasing among higher education institutions. However, MOOCs have just born and, therefore, the literature on MOOCs is scarce [4].

In this paper, we present the architecture of our MOOC called UniMOOC aemprende, a course oriented toward the fostering of entrepreneurism. Our MOOC provides students a vision of the keys to success that others entrepreneurs have applied in order to create their projects in the new digital economy.
UniMOOC aemprende is not a traditional course with a fixed calendar. Students are allowed to start at the moment they desire and to follow all the lessons available until the end of the course. UniMOOC aemprende is offering new educational content continuously, with lectures, assessment, and other activities. If a student finishes successfully the course, he or she can obtain a digital certificate or even to pass a proctor assessment to obtain a certificate issued by a university. At the moment of writing this paper, there were more than 22,000 students enrolled, and our goal is to reach no less than 40,000 students before finishing the course.

After reviewing most of the existing MOOC platforms, we chose Google CourseBuilder as the most suitable platform for the requirements of our MOOC. Besides, our MOOC makes use of different components (YouTube, Google Docs, etc.) that engage our students and help them to succeed in the course.

The rest of the paper is structured as follows. In section 2, we introduce the UniMOOC consortium and the first MOOC organized by this consortium. In section 3 , we present a brief discussion about current MOOC platforms. In section 4, we explain some details of Google CourseBuilder, the MOOC platform chosen for the development and deployment of UniMOOC aemprende. In section 5, we describe the architecture of UniMOOC aemprende, including the accreditation system compatible with Mozilla Open Badges. Finally, in section 6, we present the main conclusions.

\section{UNIMOOC}

UniMOOC aemprede ${ }^{1}$ is the first MOOC organized by UniMOOC, a consortium of Spanish universities, higher education institutions and firms interested in exploring the MOOC phenomena and promoting new methods of teaching and learning. UniMOOC was born in 2012 and it is leaded by the International Economy Institute of the University of Alicante. Besides of this, all members of UniMOOC were also interested on promoting the entrepreneurship. Because of this, UniMOOC aemprende, the first course offered by UniMOOC, is oriented toward the fostering of entrepreneurism: the theme of the course is "the keys to success in the digital economy", and the course provides students a vision of the keys that others entrepreneurs have applied in order to create their successful projects in the new digital economy.

At the beginning of the project, the first approach was to create a traditional and face-to-face course with teachers,

\footnotetext{
${ }^{1}$ http://unimooc.com/
} 
invited experts, and so. However, the audience that could be reached with this approach was very limited.

The following idea was to organize a series of lectures broadcasted online. But then, the MOOC phenomena came to change our plans: the master idea of the project was to reach as many people as possible, and the new MOOC concept was the means to this objective.

MOOCs have attracted the attention of the educational world due to the singularity of the answers they offer to common learning questions:

- They pretend to be widely open, giving the opportunity to acquire relevant knowledge without the need to spend high quantity of money on them.

- They want to reach a huge quantity of people. Some of the variables to success in MOOCs depend on the big number of student involved.

- They use the increasing number of open educational resources (OERs) and, in return, they also increase the number of OERs.

From our point of view, the main question that MOOCs pretend to solve is how to give significant and relevant education without having in count the number students.

The word "massive" in a MOOC is the keystone to this question. "Massive" configures a new scenario in which almost everything we knew about how to support online education has to be revisited and redefined.

The participants involved in the UniMOOC consortium come from different origins. Some of them are higher education institutions (mainly universities) which are specially interested in their own future. Actually, the first MOOCs were born in traditional and well recognized universities, such as Stanford University or Massachusetts Institute of Technology (MIT), leaders in the educational world. These universities started a "tsunami" [5] with potential to change the way universities approach the new education in the digital era.

Other participants of the UniMOOC consortium are firms, foundations, and non-governmental organization interested in exploring the relationship with their audience mediated by the previously mentioned higher education institutions.

In brief, all participants are exploring in a sort of "action research" method how the new models of education that MOOCs seem to provide could be a sustainable option for them and to continue developing their respective functions and services.

UniMOOC consortium has some committees to develop their functions: the steering committee, the scientific committee in charge of the development of the educational model, the technical committee in charge of the definition of the technical requirements, and the organizing committee in charge of the development of the educational resources.

The scientific committee defined the specifications that the learning platform had to accomplish. The main specifications were:
- About the educational model: It has to be based on proved and efficient didactic models. It should allow interactive improvements, but from a very simple base:

- Presenting information (based on a very carefully selection of open documents or the creation by faculty members, but trying to explore the language of communication media).

- Relevant activities in line with the information presented. All of them need to be selfevaluated activities. Some could be "stimulus" to participate in newsgroup, group activities, and other similar tasks.

- Final evaluation through automatic or peer-topeer review systems.

\section{- About the learning platform:}

- It has to support the previously mentioned educational model.

- It has to be a really open platform because it will be necessary to add new features customized to the needs of the course.

- Login with password will only be necessary to keep record of progress and to issue certifications.

- It has to be possible to access to the content used in the platform without login with password.

- It has to be scalable. The initial estimations are that in few months, the number of students enrolled will reach tens of thousands (and probably hundreds of thousands at the end of the course). Because of this, the web hosting (bandwidth, number of servers) has to be scalable to those increasing numbers.

- It has to be possible to connect with external systems for accreditation like Mozilla Open Badges to provide some "gamification" as a educational strategy. Every time the student have success passing a test it will be possible to request for an acknowledgement of this. This will contribute to motivate the student for further challenges.

- It has to allow to obtain learning analytics through the analysis of students' records.

- It has to allow to send direct e-mails to students.

- It has to be easy to use. It is foreseeable that students enrolled in UniMOOC aemprende will have not deep technical skills apart of a regular use of the Internet.

- It has to allow three different "departments" to work together at the same time: content department (educational team), secretariate (user attention and promotion tasks), and technical department (development and support of the platform).

\section{MOOC PLATFORMS}

Before developing UniMOOC aemprende, different MOOC platforms were reviewed in order to choose the most suitable for our requirements. The current available platforms aimed to develop MOOCs were reviewed based on different dimensions: 
- Open source o proprietary.

- Cloud computing or traditional web hosting.

- $\quad$ Software provided or software as a service.

On the one hand, there are some online education websites, such as Coursera, edX, and Udacity, that offer many MOOCs. Unfortunately, these websites do not provide their software, courses have to be hosted in their platforms, and the offering of a course must be negotiated with those platforms (and a contract must be signed).

On the other hand, other online education websites, such as P2PU (based on Lernata), Canvas Network (based on Canvas), and CourseSites (basado on Blackboard) are similar to Coursera, edX, and Udacity, but they allow higher education institutions to offer MOOCs without any cost. The publication of courses is almost immediate and they do not impose strong restrictions.

On the other hand, some popular LMS (Learning Management Systems), such as Moodle or Sakai, have been used to provide MOOCs. However, these LMS present some scalability problems because they were not designed to support thousands of students at the same time.

Fortunately, a new breed of LMS has appeared in recent months with the aim of providing tools to create MOOCs: edX, OpenMOOC, and Google CourseBuilder.

edX is based on XBlock, a component architecture that enables developers to create independent course components, or XBlocks [6]. The creation of a MOOC is made with the combination of XBlocks from a variety of sources, such as, text, video, wiki, and so on. XBlock was released in March 2013, so it could not be an option because it was not available at the beginning of UniMOOC aemprende.

OpenMOOC is an "an open access platform based on free source components to build a connectivist environment" [7]. We did not choose OpenMOOC for our MOOC because the identity and access management was not prepared to accept standard user accounts, such as Google, Facebook, or Twitter.

Therefore, after reviewing all existing options, we chose Google CourseBuilder as the most suitable MOOC software for the requirements of our MOOC. In the following section, the main features of Google CourseBuilder are described.

\section{COURSEBUILDER}

Google CourseBuilder ${ }^{2}$ (GCB) is an open source project of Google, not a Google product. GCB offers a lightweight way to publish course material online, track student engagement, and evaluate students' performance. An international community of GCB users is growing rapidly. GCB is a suitable solution for institutions looking for an in-house platform. Besides, as the source code is available and can be modified, institutions can develop their own customizations.

GCB's website offers a lot of information for MOOC authors. It is worth noting the section on evaluating course efficacy. The documentation explains how to collect and analyze data of various sorts.

\footnotetext{
${ }^{2}$ https://code.google.com/p/course-builder/
}

A key factor in the deployment and success of a MOOC is the scalability. Some systems start to work slowly when the number of users increases. Scalability is the ability of a system to handle a growing amount of users (workload) in a capable manner because it can be enlarged to accommodate that growth. A system without this ability cannot be enlarged to accommodate a huge amount of users, or it can be enlarged but the cost is prohibitive because adding new resources to support new users is more expensive each time.

GCB runs on Google App Engine (GAE), Google's could computing platform for developing and hosting web applications in Google's data centers. The most important feature of GAE is the high level of scalability it offers. However, GAE has an important disadvantage: GAE is a fee service. A GAE application can consume a certain level of computing resources for free (blobstore, channels, datastore operations, mails sent, etc.), controlled by a prefixed set of limits, over a 24 hour period; once one of these resources has been depleted, the resource becomes unavailable until the resource is replenished and the application stop working. Anyway, the costs associated with the use of this service are low compared to a traditional web hosting. Therefore, the use of GCB and GAE implies a cost than must be evaluated before using this LMS.

GCB is programmed in Python language. GCB can be changed and extended easily. Besides, latest version of GCB offers the possibility of adding custom components and modules. For example, GCB documentation explains how to develop a Khan Academy module [8] to import Khan Academy exercises into GCB.

\section{ARCHITECTURE}

The core of our MOOC is based on GCB. However, the use of different additional components helped us to achieve a high flexibility, scalability, and availability. We sketch the architecture of our MOOC platform in Fig. 1. The different components of this architecture are discussed in the following subsections:

- Landing page.

- CourseBuilder platform.

- Accreditation system.

- Massive email system.

- $\quad$ Supporting services.

\section{A. Landing page}

To have a huge number of students enrolled in a MOOC is not only a possibility but a need. Only if a very high number of students enroll in a MOOC, some necessary dynamics to hold the educational model will occur. Therefore, it is very important to set up mechanisms to guarantee that the desired number of students will be reached.

In UniMOOC aemprende, the original goal was to reach no less than 40,000 students before finishing the course. Unfortunately, the main page of the course was not enough to develop a marketing strategy and attract that amount of students. The information about the course that was showed in that page was scarce and limited, so a specific "landing 


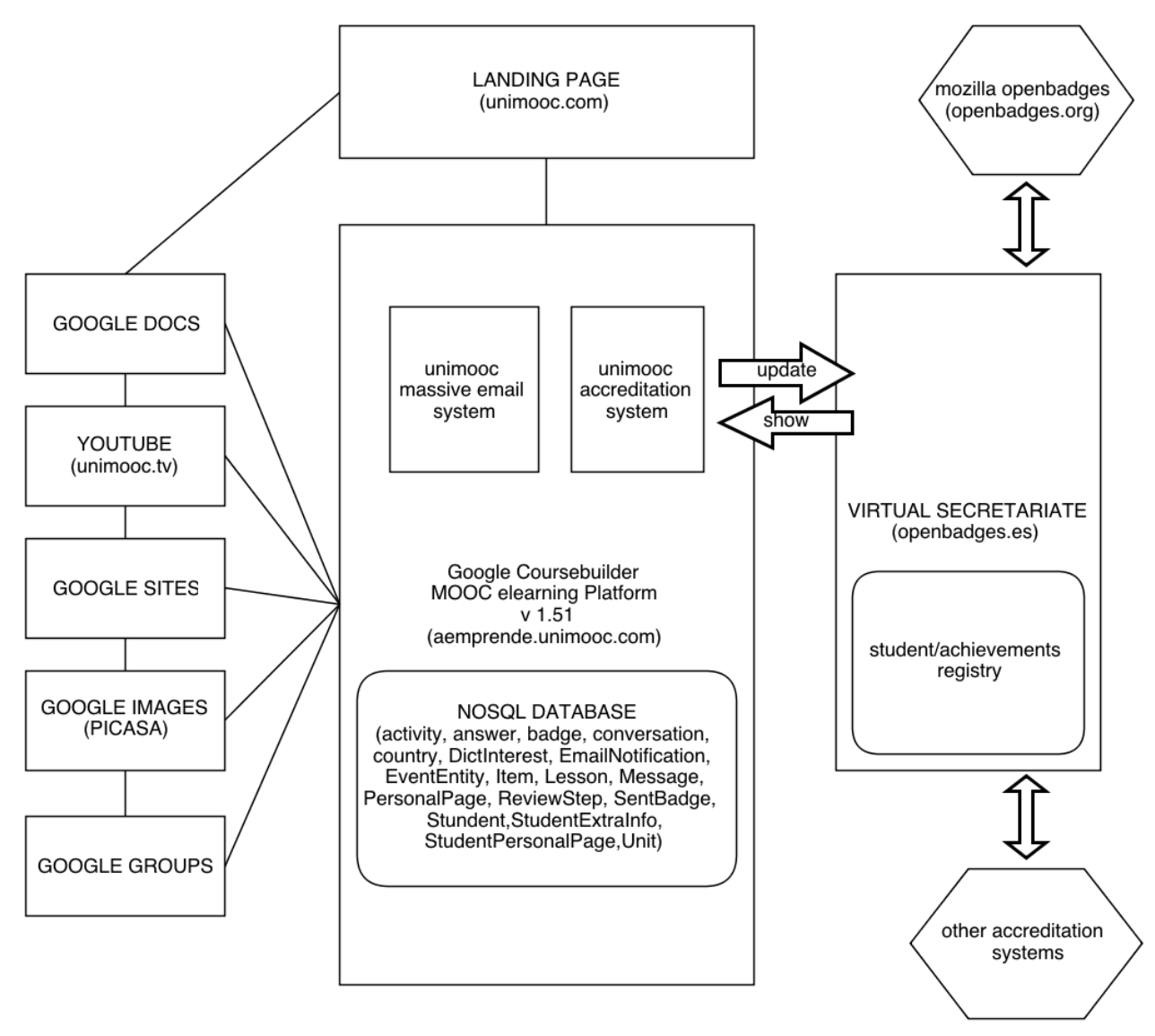

Fig. 1. Architecture of the MOOC

page" was developed in order to set up marketing campaigns and attract new students to the course.

This landing page, showed in Fig. 2, consists of a main page very carefully designed to show in a few seconds the most relevant information about UniMOOC aemprende, and some other secondary pages with additional information, such as information about partners of the project, syllabus, cooperation opportunities, and so on.

Due to some technical considerations, the landing page is not hosted in the same GCB. The landing page is hosted in a external and traditional web hosting.

\section{B. CourseBuilder platform}

GCB can be changed and extended easily. In order to fulfill the requirements of UniMOOC aemprende, some modifications and extensions that were considered essential were implemented:

- Localization and internationalization of the software to Spanish and other languages. The version 1.0 of GCB did not have this feature. Our localization and internationalization have been included in recent versions of GCB by Google.

- Creation of a more complex personal page for the students to allow them to keep record of their progress and to apply for accreditation.
- Creation of an internal social network based on sharing of interests about entrepreneurship topics.

- Creation of a component to develop the business model using the Lean Canvas methodology.

- Creation of a more complex administration page including the feature of massive email sending, statistics on-the-fly, and backups of databases for learning analytics.

- Creation of a specific accreditation module of the learning system to allow the use Mozilla Open Badges infrastructure and other similar services, including our own accreditation system.

At the present moment, UniMOOC aemprende is running the version 1.5 of GCB and all the custom features have been recoded to allow the rapid adaptation to future versions of GCB.

Two of our extensions to plain GCB have been very important for the operation of UniMOOC aemprende: the accreditation system and the massive email system. In the following subsections, these two systems are described in detail.

\section{Accreditation system}

To handle the administrative relationship about accreditation with the users, the UniMOOC developing team designed 


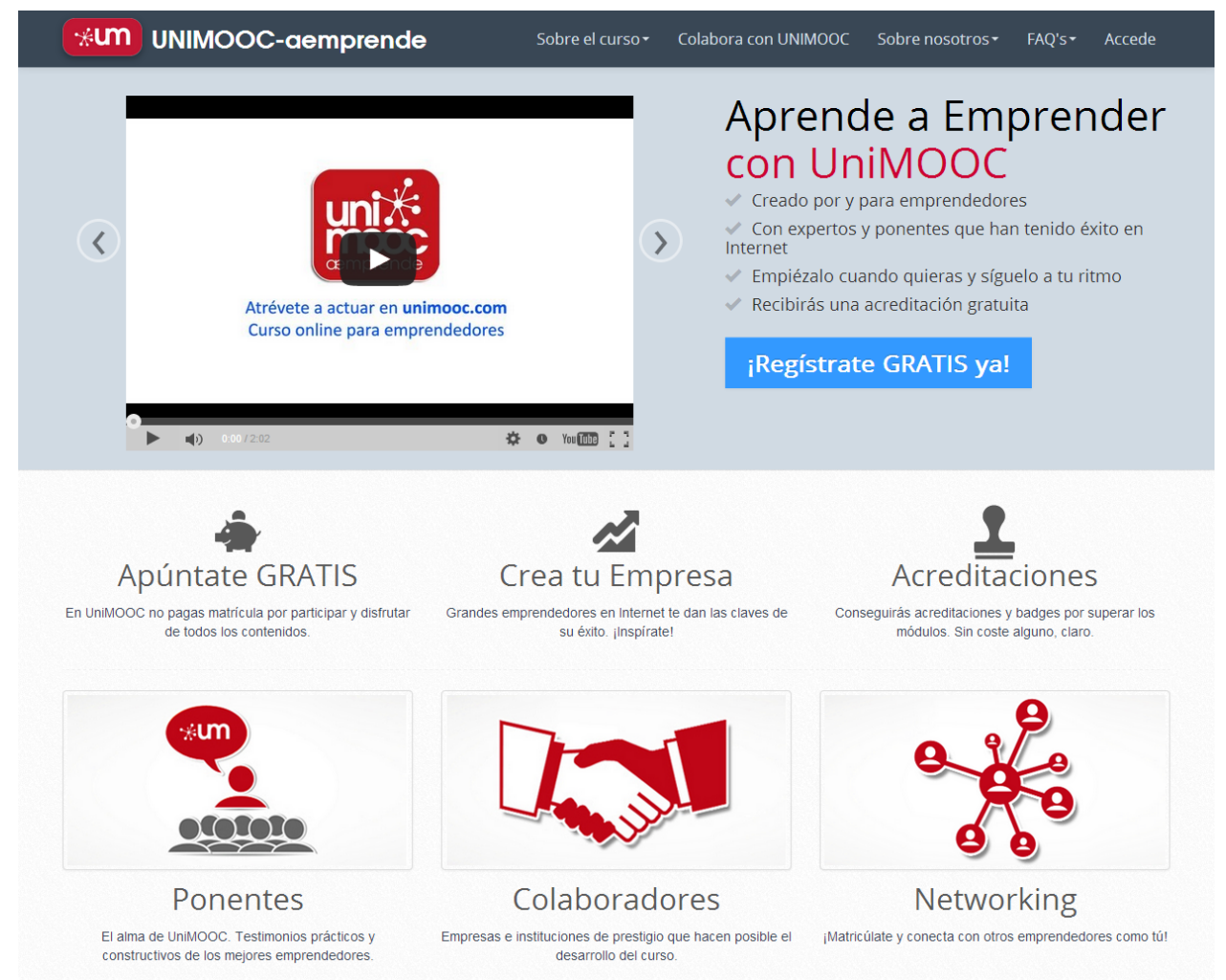

Fig. 2. Landing page of UniMOOC aemprende

a specific module to connect with the virtual secretary. This approach allows us to externalize the possibility of obtaining an accreditation of the learning progress. Therefore, different accreditation formats can be used in the same course.

At the present moment, there exists two ways of obtaining accreditations:

- $\quad$ By using Mozilla Open Badges infrastructure.

- By using the UniMOOC aemprende accreditation model.

Both methods are used to offer students a recognition of their advancement in the course.

In order to support the accreditation, instead of creating an accreditation system inside GCB itself, we designed a module that communicates to an external accreditation system that keeps a record of student's achievements. Thanks to this approach, it is possible to obtain a copy of the accreditations even in the event that the GCB of UniMOOC aemprende would be turned off in the future.

The accreditation system has two components:

- The external service in the virtual secretary.

- The internal accreditation module in GCB.

The accreditation module in GCB sends messages in JavaScript Object Notation (JSON) format to the external virtual secretary with updates of the accreditation information (e.g., new badges issued). These messages include all the information needed to update a record of accomplishment or certificate with information about all the students and their achievements. These messages are encrypted for security reasons.

The external virtual secretary is hosted in a web hosting service and it can be accessed by sending messages to a web service. Also, other future courses could use the API we have developed for the same goal.

1) The virtual secretary: The virtual secretary acts as a neutral and independent record of the achievements of students. Because it is an independent system of GCB, it can be used for many courses based or not based in GCB. Currently, the virtual secretary is hosted in the domain openbadges.es.

Only authorized users can create sets of acreditations. A set of accreditations is a variable number of accreditations related with one course.

An accreditation is a record with the following information:

- Name of the accreditation.

- Issuer name.

- Date of issue.

- URL of the main page of the course.

- URL of the criteria of the accreditation: the criteria is an external web page with information about the meaning of the accreditation. Usually, it is hosted in the course web site. However, in UniMOOC aemprende we have created a set of web pages in Google Sites to keep all the information about the criteria. 


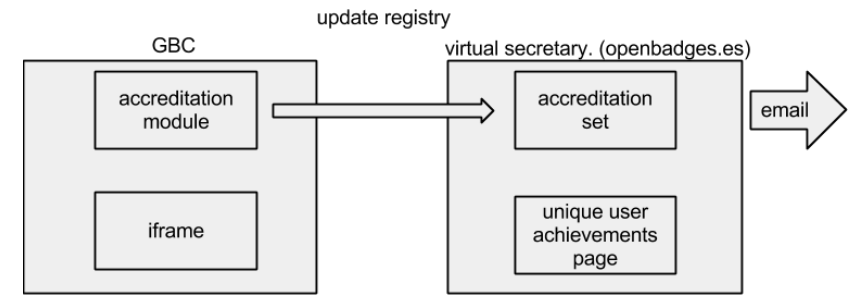

Fig. 3. Update message from the course to the virtual secretary

- URL of the image (icon) of the accreditation. This image has to follow the guidelines of the specification of Mozilla Open Badges. In UniMOOC aemprende we use Google's Picasa Web Albums to store those images.

- The list of students' emails who have earned the accreditation.

The virtual secretary provides some web services in order to:

- Update the list of students who have earned an accreditation.

- Allow students to claim a badge from Mozilla Open Badges and to include the badge in the student's Mozilla Backpack.

- Allow students to claim a specific accreditation (offered by the course administration) with all the individual accreditations aggregated.

- Check if a student has o has not right for an accreditation.

At the present moment, the virtual secretary does not provide an interface for students to directly claim for an accreditation. It only provides services to course systems connected. The course platform have to handle the relationship with their users and accreditations on their own using the web services provided by the virtual secretary.

2) The internal accreditation module in GCB: When a student passes an assessment that has an accreditation, the accreditation module in GCB sends an update message to the virtual secretary using a web service in the form of a JSON encrypted message, as it is shown in Fig. 3.

After the message has been received, decrypted, and checked, if everything is correct, the virtual secretary sends a notification message to the student with the information about the new accreditation and the instructions to claim the corresponding accreditation compatible with Mozilla Open Badges or the own UniMOOC aemprende accreditation.

Also, when students ask for a report of their progress and want to receive a specific accreditation, the GCB accreditation module connects to the virtual secretary to request the information using another web service, as it is shown in Fig. 4.

Thanks to the use of an HTML iframe, the result is displayed in the personal profile page of students in GCB. The HTML iframe that is displayed allows students to receive a digital accreditation in PDF format or to go to another personal

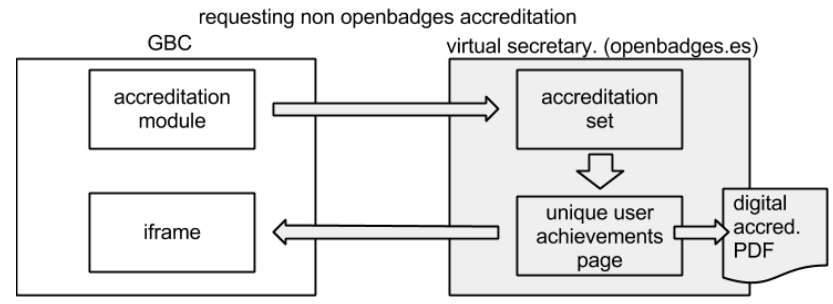

Fig. 4. Request of information about a student

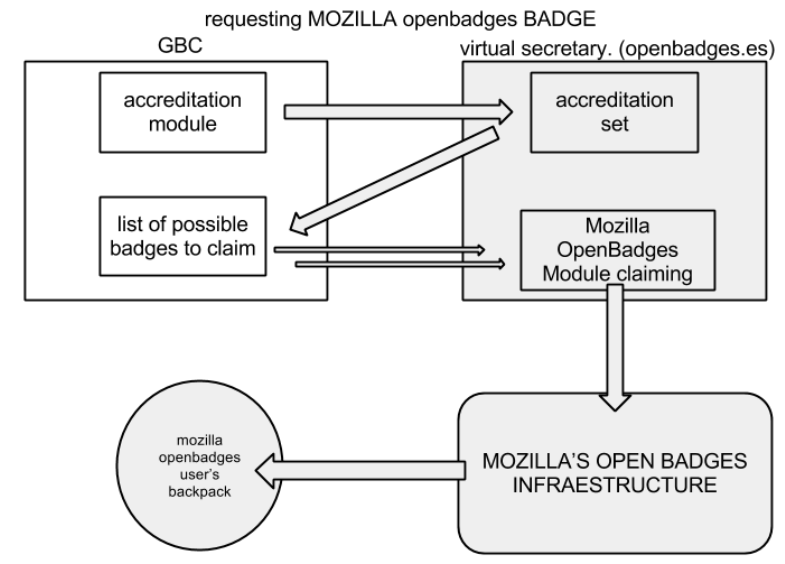

Fig. 5. Simulation Results

profile web page to request the appropriate badge compatible with Mozilla Open Badges infrastructure.

The web page that the HTML iframe displays is an unique web page for each user that shows all the accreditations that the user has earned. This page can be accessed directly using a web browser, and it is connected with the PDF accreditation through a $\mathrm{QR}$ code.

Besides of this, all the accreditations may be claimed as badges using the Mozilla Open Badges infrastructure. In that case, the GCB module displays a list of all the available achievements for a student (in the personal profile page) and links every achievement with the virtual secretary that makes possible to claim the badge compatible with Mozilla Open Badges Infrastructure.

\section{Massive email system}

GCB does not provide a notification system to directly communicate with the enrolled students. The only way is to publish an announcement in the news page in the main web page. As an alternative, the GCB developing team proposes the use of Google Groups. However, students have to sign in a specific Google Groups created by the course administrator, and since then students can receive by email the updates and news published in the Google Groups.

However, one of the requirements of UniMOOC aemprende was to directly communicate with students. During a course, different announcements can be needed to be urgently sent to students, such as the publication of new lectures.

Because of this, it was necessary to develop a custom email system to send massive emails to enrolled students. This 
system is controlled by the course administrator.

Some students can consider the emails of the course as spam. Therefore, students can choose which types of emails they want to receive in their personal home page.

Our system has some advantages and disadvantages:

- Advantages: It is possible to reach all your audience in an easy way.

- Disadvantages: There are some costs associated to this feature. The use of Google App Engine to send emails implies a cost. However, this cost is low, since it is not advisable to send more than one email per week in order to avoid to be considered as a "spammer". But even with only one email per week, some students have reported that they would prefer a lower frequency.

\section{E. Supporting services}

In UniMOOC aemprende, there exists a number of web sites or web services that provide support to the operation of the course:

- Google Drive.

- YouTube.

- Google Sites.

- Picasa Web Albums.

- Google Groups.

1) Google Drive: Google Drive (former Google Docs) is used to provide access to documents that are essential or supplemental to the video lessons of the course. After the creation of the document, full public access is given to the document. Students do not need to open an account in Google Drive, because documents are published as web pages ("publish in the Web" option). It is very important to share documents as web pages, because Google Drive has some limitations in the number of users that can access a shared document at the same time.

2) YouTube: Although the use of video lessons is strongly recommended in a MOOC, GCB does not provide any support for directly storing and broadcasting videos. Instead of this, the videos have to be broadcasted from other sources, such as Vimeo or YouTube, and then linked to the lessons in GCB.

In UniMOOC aemprende we have chosen to use YouTube to store and broadcast our videos because it offers the following benefits:

- The video hosting is decoupled from the platform.

- It is an excellent platform for the promotion of the course. The YouTube channel associated to the course has his own way to reach the audience and increases greatly the visibility of the course in search engines.

- YouTube's video editing feature covers the main needs to create good and effective videos, including subtitles, intro screens, water marks, and others features.
3) Google Sites: It is necessary to associate to the accreditation a persistent in the time document to explain the meaning of the accreditation, the value of it, and the information about the issuer of the accreditation, including links to all the necessary and supplemental information. This document should be available while the accreditation has not expired.

To guarantee these requirements, in UniMOOC aemprende we have created a set of web pages using Google Sites. Even in the event that the course is finished and the GCB is switched off, the information about the accreditations will be externally available in Google Sites.

4) Picasa Web Albums: Likewise than the videos are not stored using GCB and Google App Engine, the images files that the course uses require external hosting service to be linked.

For the image files, in UniMOOC aemprende we use the services offered by Picasa Web Albums and nowadays integrated in Google Plus. All the images that the system needs are available though this service, from logos to schemas and, of course, the images used for the accreditation system to represent the badges.

5) Google Groups: In UniMOOC aemprende, the main uses of Google Groups are:

- Announcements and optional material: for example, instructors used Google Groups to communicate reminders, announcements of new educational resources, advices to resolve commons problems, upcoming events, deadlines, etc.

- Community discussion: for students to interact with each other, asking and answering all types of questions about the course and about themes related to the course.

The creation of a community among students is very important because it can help to increase students' motivation, students can help each other (teachers and teaching assistants cannot provide support for all students in a MOOC), and a community can be used to provide extra material for students with more or less knowledge. The use of Google Groups changes the perception of the learning process: it changes from something that is perceived as individual and isolated to something that is much more social and dynamic.

However, the successful of a course community is not spontaneous. Teachers and teachers assistants have to define a strategy to promote the use of the community among students.

\section{F. Integration of the components}

One of the main requirements of UniMOOC aemprende was to create an easy to use system for students. From our personal experience, the use of different Internet services each one of them with its own login process with different user accounts is a handicap and creates severe barriers between students and their learning process.

Therefore, it is very important to make easy the access to the different contents and services of a MOOC by using the smallest possible number of user accounts. 
By using the Google ecosystem of free applications, it is possible to use the same user account for all the components of our MOOC UniMOOC aemprende:

- The main course web page.

- Google CourseBuilder.

- $\quad$ Google Drive (Google Docs).

- Google YouTube.

- $\quad$ Google Sites.

- Google Picasa Web Albums

- Google Groups.

- Google Plus.

For students, once they have logged into of any of the previous services, there is no need to $\log$ in again to access to the other services. It makes transparent and seamless for students the use of the different components of UniMOOC aemprende.

\section{CONCLUSIONS}

UniMOOC aemprende is a Massive Open Online Course (MOOC) oriented toward the fostering of entrepreneurism. UniMOOC aemprende provides students a vision of the keys to success that others entrepreneurs have applied in order to create their projects in the new digital economy.

In the core of UniMOOC aemprende's architecture is Google CourseBuilder (GCB). GCB allows us to have a full control of our MOOC. Besides, GCB is an open source project and allows us to offer content in an open way. The utilization of different online tools and services engages our students in the course and helps them to stay on track with their learning. Until now, the platform has not experienced any failure of performance or availability.

The architecture of the system has proved to be very stable and reliable. During more than 11 months, the system has been up without any problem except during maintenance scheduled events.

Only once part of the system was not available during a few hours due to external reasons. At the very beginning of the course, some files (CSS style sheets and some images) were not hosted inside GCB and Google App Engine. Instead of this, those files were stored in an external web hosting trying to optimize the use of the Google App Engine resources (the goal of this approach was to reduce the expenses of the course). Unfortunately, the external web hosting proved not to be enough powerful for a MOOC and during some hours was not responding the requests. Finally, we canceled this approach and decided to stored all the needed files to run GCB in Google App Engine itself.

The rest of the system, including YouTube, Google Drive (Docs), Google Groups, the landing page, and the virtual secretary have been always running correctly.

\section{REFERENCES}

[1] L. Pappano, "The Year of the MOOC," November 2 2012, The New York Times. [Online]. Available: http://www.nytimes.com/2012/11/04/education/edlife/massiveopen-online-courses-are-multiplying-at-a-rapid-pace.html

[2] T. L. Friedman, "Revolution Hits the Universities," January 26 2013, The New York Times. [Online]. Available: http://www.nytimes.com/2013/01/27/opinion/sunday/friedmanrevolution-hits-the-universities.html

[3] S. Kolowich, "The MOOC 'Revolution' May Not Be as Disruptive as Some Had Imagined," August 8 2012, The Chronicle of Higher Education. [Online]. Available: http://chronicle.com/article/MOOCsMay-Not-Be-So-Disruptive/140965/

[4] T. R. Liyanagunawardena, A. A. Adams, and S. A. Williams, "MOOCs: A systematic study of the published literature 2008-2012," The International Review of Research in Open and Distance Learning, vol. 14, no. 3, pp. 202-227, 2013.

[5] D. Brooks, "The Campus Tsunami," May 3 2012, The New York Times. [Online]. Available: http://www.nytimes.com/2012/05/04/opinion/brooks-the-campustsunami.html

[6] edX, "edX Takes First Step toward Open Source Vision by Releasing XBlock SDK," March 14 2013. [Online]. Available: https://www.edx.org/alert/edx-takes-first-step-toward-open/694

[7] L. Gil Sánchez and S. Martín García, "The OpenMOOC project. Platform based on free software for an open education," in Proceedings of the TERENA Networking Conference, 2013.

[8] Google, "Create Custom Modules," August 15 2013. [Online]. Available: https://code.google.com/p/course-builder/wiki/CreateModules 